\title{
Role of fibulin-3 in lung cancer: In vivo and in vitro analyses
}

\author{
SHUN XU ${ }^{1}$, YONG YANG ${ }^{2}$, YAN-BIN SUN $^{1}$, HAO-YOU WANG $^{1}$, CHANG-BO SUN $^{1}$ and XIN ZHANG ${ }^{1}$ \\ ${ }^{1}$ Department of Thoracic Surgery, The First Hospital of China Medical University; \\ ${ }^{2}$ Sujiatun Center Hospital, Shenyang, Liaoning, P.R. China
}

Received August 26, 2013; Accepted September 30, 2013

DOI: $10.3892 /$ or.2013.2799

\begin{abstract}
Lung cancer was the most commonly diagnosed cancer in 2008 worldwide. The level of fibulin-3 expression was found to be decreased in many cancer types due to aberrant promoter methylation and is correlated with poor survival of patients. However, the role of fibulin-3 and which form of fibulin-3 is expressed in lung cancer cells remain unclear. Therefore, pathologic and functional studies were carried out to determine the role of fibulin-3 in suppressing lung cancer both in vivo and in vitro. In the present study, we found that the levels of fibulin-3 mRNA and protein were lower in cancer tissues than in normal tissues. Downregulation of fibulin-3 mRNA in tumor tissues was associated with an increase in fibulin-3 promoter methylation. Circulating fibulin-3 was significantly associated with tumor progression, survival rate of lung cancer patients, and the number of circulating tumor cells (CTCs). To examine the effects of exogenous expression of fibulin-3 in vitro, lung cancer A549 cells were transfected with the pEGFP-C1-fibulin-3 expression vector. Relative to the untreated cells, fibulin-3-expressing cells exhibited lower proliferation and mobility as determined by MTT and Transwell assays, respectively. To conclude, our results suggest that fibulin-3 negatively modulates the invasiveness of lung cancer cells via regulation of $\mathrm{p} 38-\mathrm{MAPK}$ and MMP-2/9.
\end{abstract}

\section{Introduction}

Lung cancer was the most commonly diagnosed cancer as well as the leading cause of cancer-related mortality in males in 2008 worldwide (1). Similar to other cancers, lung cancer is thought to be caused by the deregulation of normal gene expression (2).

Fibulins are widely distributed and are localized at basal membranes mediating cell-to-cell and cell-to-matrix communication. They are characterized by repeated epidermal growth factor (EGF)-like domains and a unique C-terminal struc-

Correspondence to: Dr Shun Xu, Department of Thoracic Surgery, The First Hospital of China Medical University, Nanjing North Street 155, Heping, Shenyang, Liaoning 110001, P.R. China E-mail: xushun_2008@163.com

Key words: fibulin-3, lung cancer, survival analysis, prognosis, methylation ture $(3,4)$. The relationship of the fibulin gene family members and many types of cancer has been reported (5-7). The fibulin-3 gene, also known as EFEMP1 (epidermal growth factorcontaining fibulin-like extracellular matrix protein 1) located at chromosome 2p16, is one of the 7 members of the fibulin gene family of extracellular glycoproteins (8). The level of fibulin-3 expression was found to be decreased in many cancer types due to aberrant promoter methylation and is correlated with poor survival of patients with breast cancer (9) lung cancer (10) and hepatocellular carcinoma (11). Lecka-Czernik et al (12) predicted that this protein has 2 possible isoforms, a 'long' form of 53 to $55 \mathrm{kDa}$, with an $\mathrm{NH} 2$-terminal $\mathrm{Ca}^{2+}$-binding epidermal growth factor-like repeat, and a 'short' form of 40 to $43 \mathrm{kDa}$ without this domain. However, which form of fibulin-3 is expressed in lung cancer cells remains unclear.

Pathologic and functional studies were carried out to determine the role of fibulin-3 in suppressing lung cancer both in vivo and in vitro. Further elucidation of the functions of fibulin-3 may lead to a better understanding of the pathogenesis of lung cancer. Fibulin-3 is a potential molecular marker for lung cancer.

\section{Patients and methods}

Cell culture. The A549 cell line was obtained from ATCC (Rockville, MD, USA). Cells were maintained at $37^{\circ} \mathrm{C}$ in a $5 \% \mathrm{CO}_{2}$ incubator in RPMI-1640 medium (Sigma-Aldrich, Carlsbad, CA, USA) containing 10\% heat-inactivated fetal bovine serum (FBS) and 1\% penicillin-streptomycin.

Subjects. This study was approved by China Medical University Ethics Committee and was conducted according to regulations of the Helsinki Declaration. All patients provided written informed consent to participate in the present study. Fifty-six patients who were diagnosed with primary non-small cell lung cancer (NSCLC) at the Department of Thoracic Surgery, The First Affiliated Hospital of China Medical University from January 2006 to December 2010 were included in the present study. Twenty-eight patients had stage IIIB NSCLC (locally advanced) and 28 patients had stage IV NSCLC (metastatic) according to the International Association for the Study of Lung Cancer (IASLC) staging committee (13). All diagnoses were based on standard laboratory tests (cytology and histology), and confirmed by computerized tomography of the thorax. None of the patients underwent radiotherapy or chemotherapy prior to surgery. 
Quantitative real-time PCR. Total RNA was isolated from the tissues using TRIzol reagent (Invitrogen Life Technologies, Carlsbad, CA, USA) according to the manufacturer's protocol. First-strand cDNA was reverse transcribed with $1 \mu \mathrm{g}$ of total RNA, using Takara Reverse Transcription kit and oligo(dT)15 primers (both from Takara, Dalian, China). The resultant cDNA was then used for quantitative PCR reactions. The fibulin-3 primers were: 5'-GAGCAGCTCCAGGGGACCGCCGCG-3' (sense) and 5'-TCCCCGACACGCTACCTTCG-3' (antisense). The housekeeping gene, $G A P D H$, was used as an internal control for normalization of the results. The GAPDH primers were: 5'-AGAAGGCTGGGGCTCATTTG-3' (sense) and 5'-AGGGGCCATCCACAGTCTTC-3' (antisense). Amplification of fibulin-3 and GAPDH was performed with 1 cycle at $95^{\circ} \mathrm{C}$ for $10 \mathrm{~min}$, and 40 cycles at $95^{\circ} \mathrm{C}$ for $15 \mathrm{sec}$ and $60^{\circ} \mathrm{C}$ for $60 \mathrm{sec}$. Calculation of the relative expression of each transcript was performed using the $2^{-\Delta \Delta C t}$ method.

Methylation-specific PCR (MSP). Genomic DNA was extracted from lung cancer specimens using a TissueGen DNA kit (CWbiotech, Beijing, China). Genomic DNA $(2 \mu \mathrm{g})$ was denatured with $0.2 \mathrm{M} \mathrm{NaOH}$. Then, $10 \mathrm{mM}$ hydroquinone and $3 \mathrm{M}$ sodium-bisulfite (both from Sigma) were added. The solution was incubated at $55^{\circ} \mathrm{C}$ for $16 \mathrm{~h}$. DNA samples were then purified using a Wizard ${ }^{\circledR}$ DNA Purification Resin (Promega Corporation, Madison, WI, USA). In this procedure unmethylated (but not methylated) cytosines are converted to uracil, which is then converted to thymidine during subsequent PCR to give sequence differences between methylated and unmethylated DNA. The modified DNA was used as a template both for MSP and USP. The primer sequences for the methylated fibulin-3 gene were: 5'-GTAGTTTTAGGGGATCGTCGC-3' (sense) and 5'-TCCCCGACACGCTACCTTCG-3' (antisense); and for the unmethylated allele were: 5'-GAGTAGTTTTAGG GGATTGTTGT-3' (sense) and 5'-TCCCCAACACACTACCT TCA-3' (antisense). The PCR products were separated on $2 \%$ agarose gel with ethidium bromide and visualized under UV illumination.

Western blot analysis. The tissues or cells were lysed in lysis buffer (20 mM Tris-HCl, $150 \mathrm{mM} \mathrm{NaCl}, 2$ mM EDTA, 1\% Triton X-100) containing a protease inhibitor cocktail (Sigma). The extracted protein amounts were quantified using the BCA protein assay kit (CWbiotech). Equivalent amounts of protein $(40 \mu \mathrm{g})$ were separated using $10 \%$ SDS-PAGE and transferred to a PVDF membrane (Millipore, Billerica, MA, USA). Western blotting was performed using the following primary antibodies: fibulin-3 (sc-365224), MMP-2 (sc-8835), MMP-9 (sc-12759), phospho-JNK (sc-12882), p38 (sc-7149), phospho-p38 (sc-166182), ERK 1/2 (sc-135900), phosphoERK 1/2 (sc-16982) and $\beta$-actin (sc-130657) (all from Santa Cruz Biotechnology, Inc., Santa Cruz, CA, USA). The binding for each specific antibody was detected with horseradish peroxidase (HRP)-conjugated respective secondary antibodies and ECL solutions (both from Amersham Biosciences, Amersham, UK).

Immunohistochemical staining. Tissues were fixed with $10 \%$ buffered formalin, embedded in paraffin, and decalcified in
$10 \%$ EDTA solution. Representative blocks were then cut into 4- $\mu \mathrm{m}$ sections, deparaffinized with xylene and rehydrated in a series of ethanol washes $(100,90,80$ and $70 \%)$. The sections were then incubated with $3 \% \mathrm{H}_{2} \mathrm{O}_{2}$ and $5 \%$ serum to block endogenous peroxidase activity and non-specific binding. For the fibulin-3 protein, sections were incubated with the antihuman fibulin-3 antibody. The sections were then incubated with biotinylated secondary antibodies and visualized by DAB. Counterstaining was carried out with hematoxylin. The sections were dehydrated in alcohol and coverslipped. For the negative controls, PBS replaced the primary antibody.

Enzyme-linked immunosorbent assay. Levels of fibulin-3 were measured in peripheral blood of the patients with NSCLC and quantified in nanograms/milliliter with the use of the human fibulin-3 enzyme-linked immunosorbent assay (Uscn Life Science Inc., Wuhan, China).

Isolation and enumeration of circulating tumor cells (CTCs). Blood samples $(7.5 \mathrm{ml})$ from the patients with NSCLC were drawn into CellSave ${ }^{\circledR}$ tubes (Veridex LLC, Warren, NJ, USA), which were maintained at room temperature (RT) and processed within $72 \mathrm{~h}$ of collection. CTCs were defined as nucleated EpCAM-positive cells, lacking CD45 but expressing cytoplasmic cytokeratins 8, 18 and 19. All CTC evaluations were performed by qualified and trained personnel.

Transient transfection. The plasmid pEGFP-C1-fibulin-3 (gifted by Li Yin, China Medical University) was transfected into A549 cells by Lipofectamine ${ }^{\mathrm{TM}} 2000$ (Invitrogen Life Technologies) according to the manufacturer's protocol. Twenty four hours post-transfection, drug-resistant clones were isolated and expanded. A549 cells transfected with pEGFP-C1 were used as a control. All gene expression studies were conducted using pools of colonies $(n=150)$ to avoid a clonal bias.

Immunofluorescence. Cells were washed with PBS, fixed in $4 \%$ paraformaldehyde, permeabilized in 1\% Triton X-100 for 5 min, and blocked with $5 \%$ bovine serum albumin (BSA) in PBS containing $0.5 \%$ Triton $\mathrm{X}-100$ for $1 \mathrm{~h}$. Fibulin-3 expression was detected using the anti-fibulin-3 antibody for $1 \mathrm{~h}$ at room temperature. Cells were washed with PBS and incubated with the appropriate secondary fluorophore-conjugated antibody for $1 \mathrm{~h}$ at RT, washed with PBS, and mounted using SlowFade ${ }^{\circledR}$ Gold Antifade reagent (Invitrogen Life Technologies). The secondary antibody used for detection of fibulin-3 was Alexa Fluor ${ }^{\circledR} 594$ rat anti-mouse IgG $(\mathrm{H}+\mathrm{L})$ (Invitrogen Life Technologies).

MTT assay. Cells were plated in 96-well plates (1,500 cells/well). After $24 \mathrm{~h}, 0.5 \mathrm{mg} / \mathrm{ml} \mathrm{3-(4,5-dimethylthi-}$ azol-2-yl)-2,5-diphenyl tetrazolium bromide (MTT; Sigma) was added to each well. Four hours later, cells were lysed with dimethyl sulfoxide (DMSO), and the absorbance rates were measured at 550-560 nm using a microplate reader (Bio-Rad, Hercules, CA, USA).

In vitro wound healing assay. Cells were grown in a 6-well dish. A confluent monolayer of cells was scratched with a $200-\mu 1$ pipette tip to simulate a wound. Cells were washed 
$\mathbf{A}$

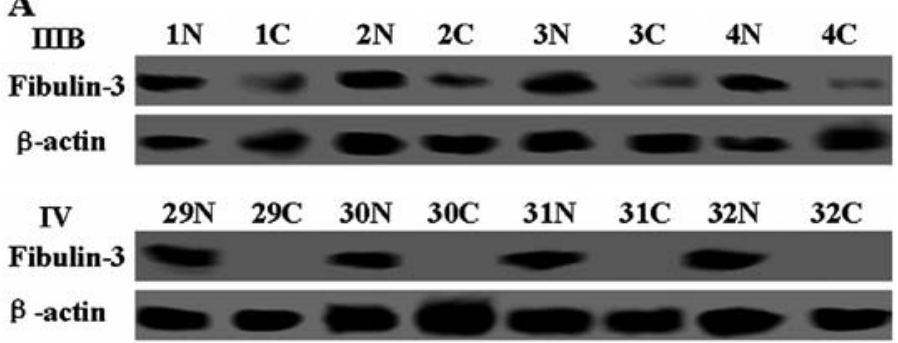
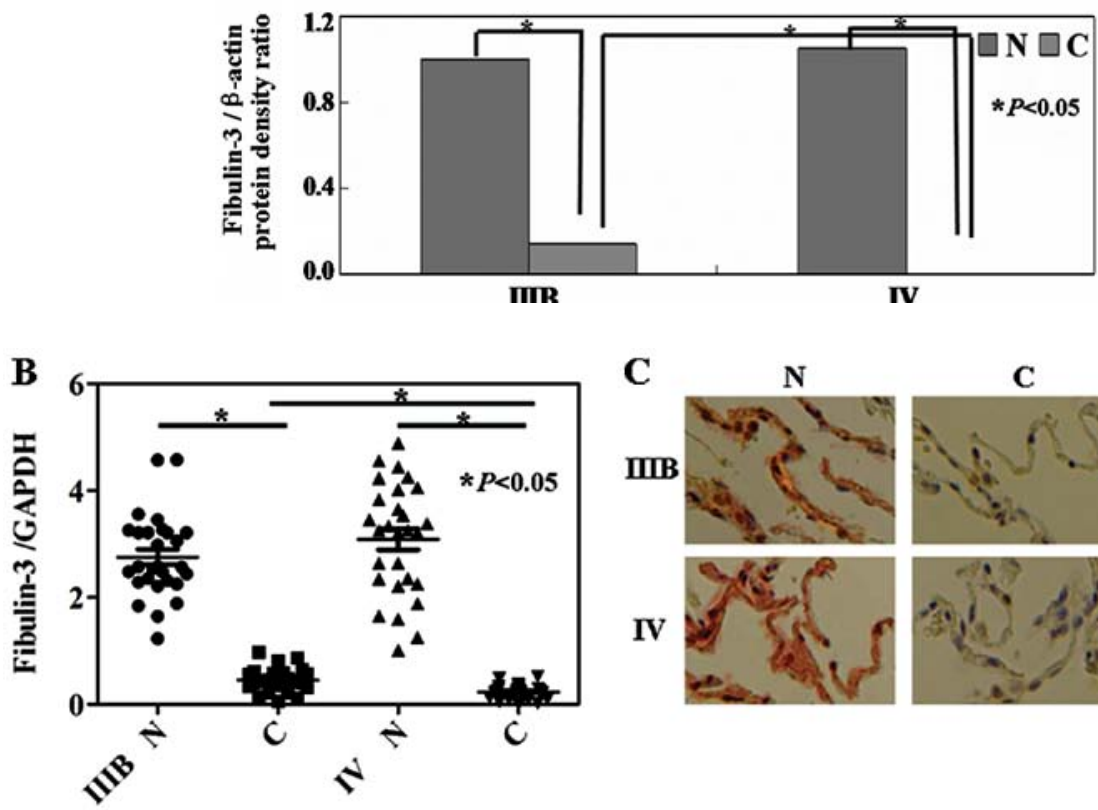

C
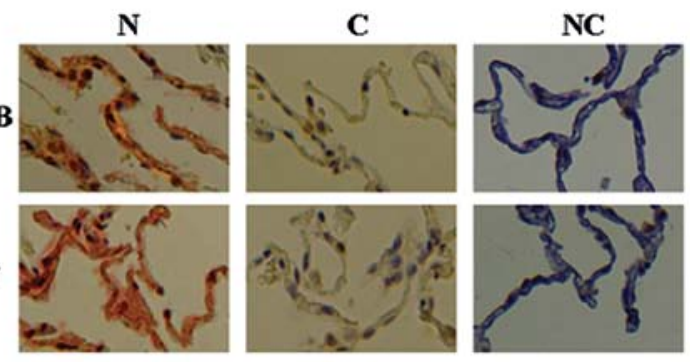

D

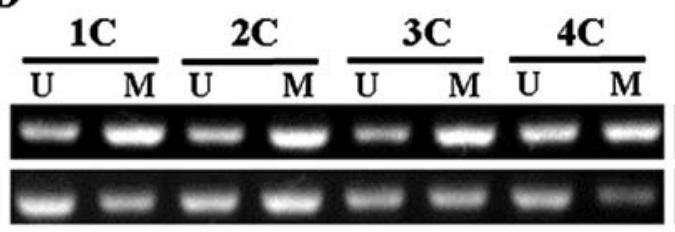

$\frac{29 \mathrm{C}}{\mathrm{U} M} \frac{30 \mathrm{C}}{\mathrm{U} M} \frac{31 \mathrm{C}}{\mathrm{U} M} \frac{32 \mathrm{C}}{\mathrm{U} M}$
$-\infty-\infty$

Figure 1. (A) Representative results of fibulin-3 expression in 4 paired lung cancer and corresponding normal tissues by western blotting. $\beta$-actin was used as an internal control. (B) The levels of fibulin-3 mRNA were measured in specimens using real-time PCR. GAPDH was used as an internal control. (C) Immunohistochemical staining for fibulin-3 protein in the specimens. The nuclei were counterstained with hematoxylin. (D) Methylation-specific PCR (MSP) analysis of CpG island methylation within the fibulin-3 promoter region in the specimens. PCR products specific for unmethylated (U) and methylated (M) sites. CpG sites were analyzed using electrophoretic separation on a $2 \%$ agarose gel. $\mathrm{N}$, normal; $\mathrm{C}$, cancer; NC, negative control.

twice with PBS and then supplemented with medium and incubated for $4 \mathrm{~h}$ at $37^{\circ} \mathrm{C}$. Cell migration into the wounded area was monitored microscopically. Images were captured at the interface of the unwounded and wounded areas.

Transwell migration assay. Cells were plated at $2 \times 10^{5}$ cells/well in $0.5 \mathrm{ml}$ of serum-free medium in 24-well Matrigel-coated Transwell units with polycarbonate filters $(8-\mu \mathrm{m}$ pore size, Costar Inc., Milpitas, CA, USA). The lower chamber was loaded with $600 \mu \mathrm{l}$ of RPMI-1640 containing 10\% FBS. After incubation for $24 \mathrm{~h}$ in normal culture conditions, we did not see any cells floating in the upper chamber, indicating that the cells had not undergone apoptosis at this time-point. The top surface of the membrane was gently scrubbed with a cotton bud and fixed in 4\% paraformaldehyde (Sigma) and stained with crystal violet. The cells that had invaded through the membrane filters were counted using a light microscope. Ten microscopic fields (x400) were randomly selected to count the cells.

Statistical analysis. All experiments were performed in triplicate, and the results are expressed as the means \pm standard deviation (SD). Kaplan-Meier survival plots were generated and comparisons were made with log-rank statistics. P-values $<0.05$ were considered to indicate statistically significant results. All the statistical analyses and graphics were performed with GraphPad Prism version 5.00 for Windows (GraphPad Software, San Diego, CA, USA).

\section{Results}

Detection of fibulin-3 mRNA and protein levels in lung tissue samples. Real-time PCR and western blot assays were 

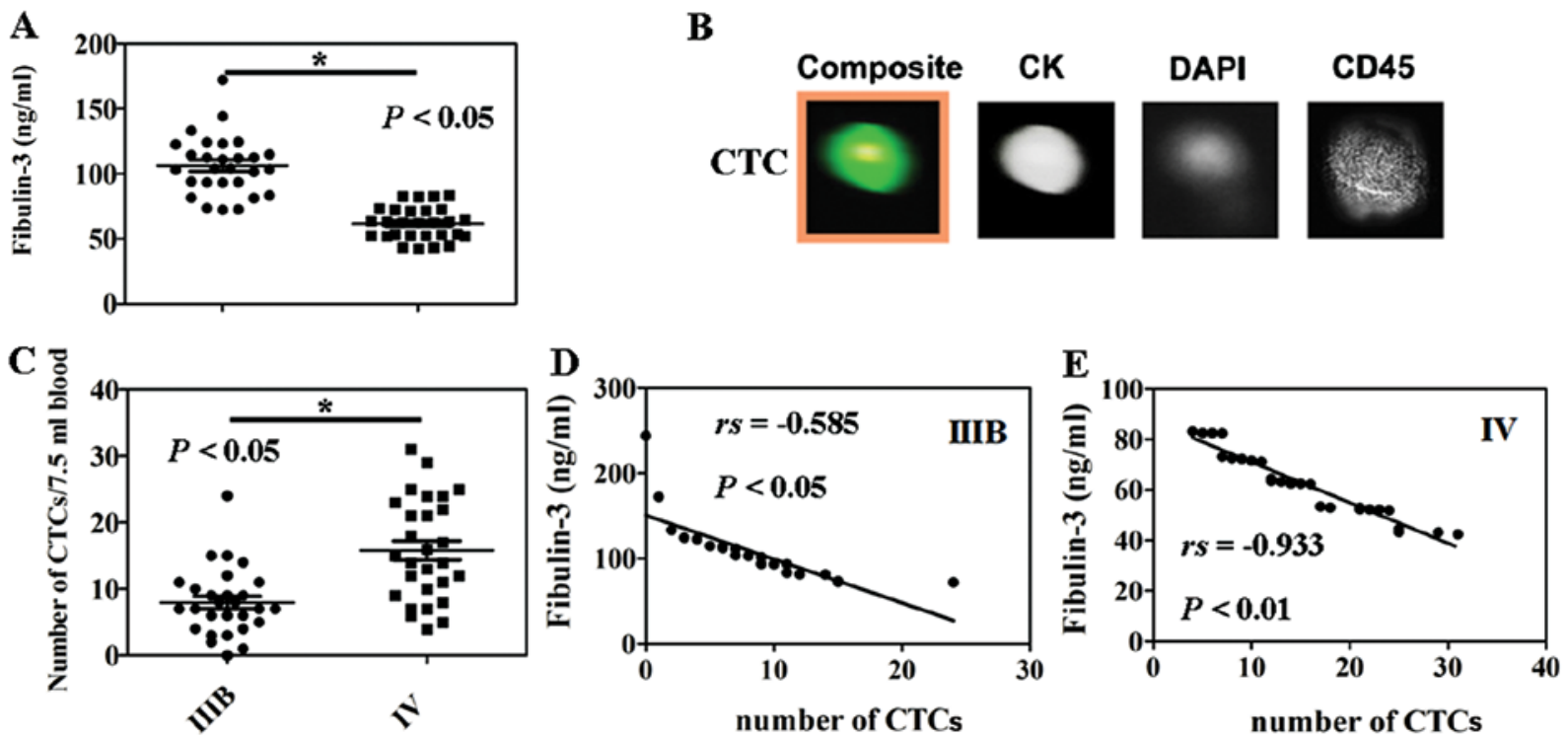

Figure 2. (A) Plasma fibulin-3 levels were detected using ELISA. There was a significant difference in plasma fibulin-3 level between the patients with stage IIIB NSCLC and the patients with stage IV NSCLC (P<0.05). (B) CellSearch ${ }^{\circledR}$ images of CTCs from patients with NSCLC. (C) Circulating tumor cells (CTCs) in the serum of patients with stage IIIB and stage IV NSCLC were isolated by using the CellSearch ${ }^{\circledR}$ system. There was a negative correlation between serum levels of fibulin-3 and CTCs in the patients with (D) stage IIIB and (E) stage IV NSCLC. NSCLC non-small cell lung cancer.
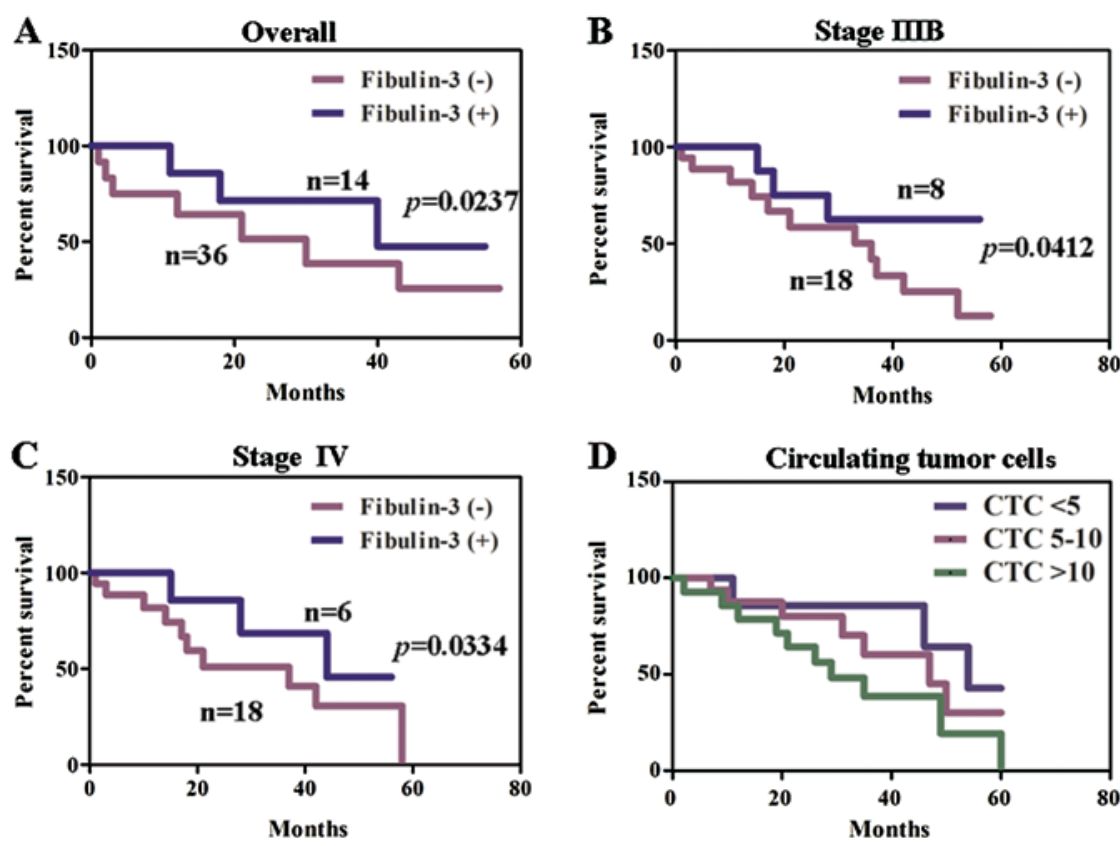

Figure 3. Kaplan-Meier survival curves of (A) all patients and patients with (B) stage IIIB (C) stage IV NSCLC according to serum fibulin-3 level. (D) Kaplan-Meier plot estimating overall survival for 3 risk groups according to CTCs in plasma ( $<5$ CTCs, 5-10 CTCs, $>10$ CTCs). All results were statistically significant $(\mathrm{P}<0.05)$. NSCLC non-small cell lung cancer. CTCs, circulating tumor cells.

performed to detect the levels of fibulin-3 mRNA and protein. As shown in Fig. 1A and B, the levels of fibulin-3 mRNA and protein were low in the low-stage NSCLC (IIIB) tissues and undetectable in the advanced stage (IV) tissues $(\mathrm{P}<0.05)$. Immunohistochemistry was then performed on 56 NSCLC tissue samples and the corresponding non-tumor tissue samples. Representative examples of fibulin-3 protein expression in NSCLC tissue samples showed that fibulin-3 protein was weakly expressed in the lung cancer specimens, but was highly expressed in the normal areas of the specimens (Fig. 1C). A correlation was noted between fibulin-3 promoter methylation and downregulation of fibulin-3 mRNA levels in the tumor samples. Methylated tumor lung tissue samples showed loss of fibulin-3 mRNA (Fig. 1D). Taken together, the loss of fibulin-3 gene expression in NSCLC may be correlated with the methylation of the fibulin-3 gene promoter.

Correlation of fibulin-3 serum levels and CTCs. A statistically significant difference was observed between circulating fibulin-3 levels in the low stage NSCLC (IIIB) patients and the 
Table I. Correlation between fibulin-3 expression and clinicopathological parameters of the patients with stage IIIB and IV NSCLC.

\begin{tabular}{|c|c|c|c|c|c|c|c|c|c|c|c|c|}
\hline \multirow{2}{*}{$\begin{array}{l}\text { Clinicopathological } \\
\text { features }\end{array}$} & \multicolumn{6}{|c|}{ Fibulin-3 expression (IIIB) } & \multicolumn{6}{|c|}{ Fibulin-3 expression (IV) } \\
\hline & $\mathrm{n}$ & - & + & PR $(\%)$ & $\chi^{2}$ & $\mathrm{P}$-value & $\mathrm{n}$ & - & + & $\mathrm{PR}(\%)$ & $\chi^{2}$ & P-value \\
\hline Gender & & & & & 0.69 & 0.406 & & & & & 1.70 & 0.192 \\
\hline Female & 8 & 4 & 4 & 50.0 & & & 10 & 6 & 4 & 40.0 & & \\
\hline Male & 20 & 15 & 5 & 25.0 & & & 18 & 16 & 2 & 11.1 & & \\
\hline Age (years) & & & & & 0.00 & 0.976 & & & & & 0.05 & 0.827 \\
\hline$<50$ & 11 & 8 & 3 & 27.3 & & & 8 & 6 & 2 & 25.0 & & \\
\hline$\geq 50$ & 17 & 11 & 6 & 35.3 & & & 20 & 16 & 4 & 20.0 & & \\
\hline Differentiation & & & & & 0.12 & 0.734 & & & & & 1.85 & 0.173 \\
\hline Well or moderate & 9 & 6 & 3 & 33.3 & & & 6 & 3 & 3 & 50.0 & & \\
\hline Poor & 19 & 13 & 6 & 31.6 & & & 22 & 19 & 3 & 13.6 & & \\
\hline Lymphatic invasion & & & & & 0.00 & 1.00 & & & & & 0.18 & 0.673 \\
\hline- & 14 & 9 & 5 & 35.7 & & & 19 & 14 & 5 & 26.3 & & \\
\hline+ & 14 & 10 & 4 & 28.6 & & & 9 & 8 & 1 & 11.1 & & \\
\hline Venous invasion & & & & & 1.13 & 0.287 & & & & & 0.38 & 0.536 \\
\hline- & 12 & 6 & 6 & 50.0 & & & 18 & 13 & 5 & 27.8 & & \\
\hline+ & 16 & 13 & 3 & 18.8 & & & 10 & 9 & 1 & 10.0 & & \\
\hline Histological type & & & & & 0.23 & 0.891 & & & & & 0.01 & 0.905 \\
\hline Squamous cell & 9 & 6 & 3 & 33.3 & & & 9 & 7 & 2 & 22.2 & & \\
\hline Adenocarcinoma & 8 & 5 & 3 & 37.5 & & & 9 & 7 & 2 & 22.2 & & \\
\hline Small cell & 11 & 8 & 3 & 27.3 & & & 10 & 8 & 2 & 20.0 & & \\
\hline Tumor size $(\mathrm{cm})$ & & & & & 0.07 & 0.794 & & & & & 0.02 & 0.893 \\
\hline$<3$ & 15 & 11 & 4 & 26.7 & & & 17 & 14 & 3 & 17.6 & & \\
\hline$\geq 3$ & 13 & 8 & 5 & 38.5 & & & 11 & 8 & 3 & 27.3 & & \\
\hline pN category & & & & & 0.19 & 0.979 & & & & & 1.38 & 0.713 \\
\hline $\mathrm{pN} 0$ & 6 & 4 & 2 & 33.3 & & & 6 & 5 & 1 & 16.7 & & \\
\hline $\mathrm{pN} 1$ & 7 & 5 & 2 & 28.6 & & & 7 & 5 & 2 & 28.6 & & \\
\hline $\mathrm{pN} 2$ & 8 & 5 & 3 & 37.5 & & & 6 & 4 & 2 & 33.3 & & \\
\hline $\mathrm{pN} 3$ & 7 & 5 & 2 & 28.6 & & & 9 & 8 & 1 & 11.1 & & \\
\hline
\end{tabular}

NSCLC non-small cell lung cancer; PR, positive rate; $\chi^{2}$, Chi-square distribution.

advanced stage (IV) patients $(\mathrm{P}<0.05$, Fig. 2A). In the present study, we isolated and enumerated the CTCs in patients using the CellSearch ${ }^{\circledR}$ system (Fig. 2B). Less CTCs were noted in the peripheral blood of patients with low stage NSCLC (IIIB) when compared with the patients with advanced stage NSCLC (IV) ( $\mathrm{P}<0.05$, Fig. 2C). The plasma levels of fibulin-3 and the number of CTCs were significantly negatively correlated between stage IIIB and IV NSCLC patients $(r=-0.585$, $\mathrm{P}<0.05$, Fig. $2 \mathrm{D}$ or $\mathrm{r}=-0.933, \mathrm{P}<0.05$, Fig. $2 \mathrm{E})$, respectively.

Fibulin-3 and clinicopathological variables. We analyzed the potential relationship between the expression of fibulin-3 and the clinicopathological characteristics of the NSCLC patients. Fibulin-3 expression was not associated with any of the clinicopathological characteristics of the patients with stage IIIB or IV NSCLC (Table I). However, the serum level of fibulin-3 was associated with lymphatic invasion $(\mathrm{P}<0.05$, Table II). To investigate the correlation of serum level of fibulin-3 with patient survival, the survival data from 50 patients with NSCLC (6 missing at follow-up) were assessed. In patients with stage IIIB and IV NSCLC, comparison by the Kaplan-Meier method according to low vs. high fibulin-3 expression showed a significant difference in the 5 -year survival rate $(\mathrm{P}<0.05$, Fig. $3 \mathrm{~A}-\mathrm{C})$. The patients with $<5$ CTCs showed a higher survival rate when compared with the patients with 5-10 CTCs or with >10 CTCs. A significantly different survival rate was confirmed between the patients with 5-10 CTCs and the patients with >10 CTCs in the Cox model $(\mathrm{P}<0.05$, Fig. 3D).

Effect of fibulin-3 expression on the proliferation and migratory capacity in A549 cells. A549 cells were transfected with the pEGFP-C1-fibulin-3 expression vector, and the levels of fibulin-3 protein and mRNA were measured by western blotting, RT-PCR and immunofluorescence. As shown in Fig. 4A, the results of RT-PCR and western blot analysis confirmed exogenous expression of fibulin-3 in A549 cells after 

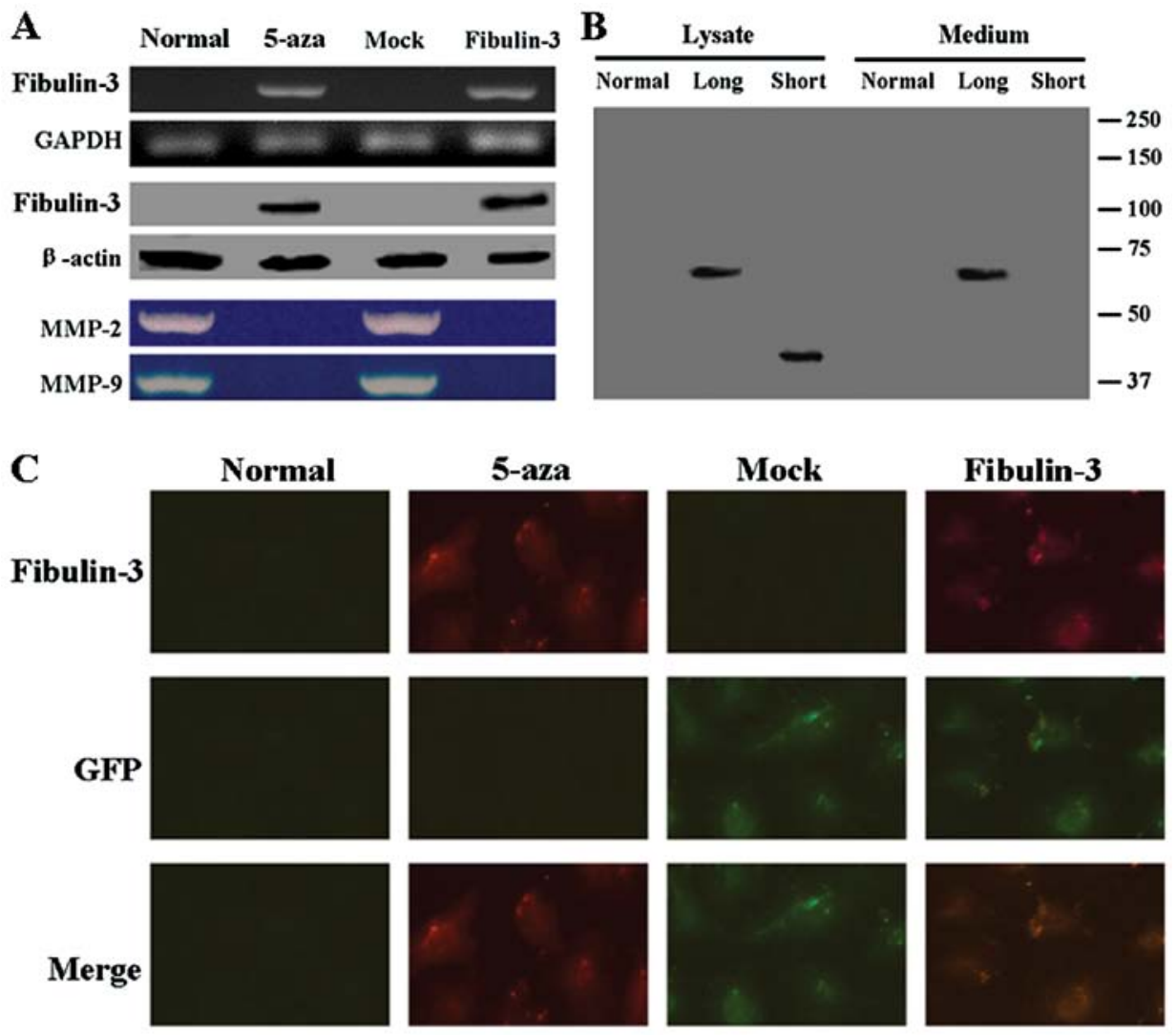

Figure 4. (A) Fibulin-3 mRNA and protein levels in A549 cells after transfection with pEGFP-C1-fibulin-3 were detected using RT-PCR and western blotting, respectively. Gelatinolytic activity of secreted MMP-2 and -9 in A549 cells was analyzed by zymography. (B) Recombinant short form of fibulin-3 was produced much less efficiently than the long form of fibulin-3 and was secreted at very low or undetectable amounts in the culture medium. (C) Detection of fibulin-3 and GFP in transfected and untransfected A549 cells by immunofluorescence. Normal, untreated A549 cells; mock, A549 cells transfected with pEGFP-C1; 5'-aza, 5'-aza-2'-deoxycytidine.

transfection. Immunofluorescence analysis showed the localization of GFP and fibulin-3 in the fibulin-3-expressing A549 cells (Fig. 4C).We observed that the long form of fibulin-3 was largely secreted into the culture medium, whereas the short form was expressed at lower levels, accumulated in the total cell lysate, and was virtually absent from the culture medium (Fig. 4B). We next assessed the effect of fibulin-3 expression on the growth of A549 cells. The growth curves obtained by MTT assays demonstrated that fibulin-3 inhibited the growth of A549 cells ( $\mathrm{P}<0.05$, Fig. $5 \mathrm{~A})$. Next, the mobility of fibulin-3-expressing A549 cells was determined using wound-healing and Transwell assays. As shown in Fig. 5B, the percentage of wound closure of A549 cells (16.7\%) was decreased when compared to the untreated $(100.0 \%)$ and mock transfected $(100.0 \%)$ cells $(\mathrm{P}<0.05)$. Overexpression of the fibulin-3 gene significantly inhibited the invasion of A549 cells into the Matrigel as detected using Transwell assay $(\mathrm{P}<0.05$, Fig. 5C). Furthermore, the activities of MMP-2 and -9 were significantly decreased in cells transfected with pEGFPC1-fibulin-3 when compared to the untreated cells (Fig. 4A).

Fibulin-3 suppresses the p38/MAPK signaling pathway. To identify the mechanism of fibulin-3, western blot assays were performed to detect changes in possible signaling pathway proteins. Expression of MMP-2 and -9 was downregulated in fibulin-3-expressing A549 cells. While total levels of p38 and
ERK1/2 showed no changes, the levels of phospho-p38 and phospho-ERK1/2 were observed to be significantly higher in the fibulin-3-positive cells when compared to that in negative cells (Fig. 6). Notably, phospho-JNK was also increased in the fibulin-3-positive cells (Fig. 6). In combination, these results suggest that fibulin-3 inhibited the mobility of A549 cells via the p38 and ERK 1/2 proteins.

\section{Discussion}

Fibulin-3 has been found to be downregulated in several types of solid tumors (14) but surprisingly, is the most upregulated member of the fibulin family in malignant gliomas (15). Fibulin-3 is also upregulated in aggressive pancreatic adenocarcinoma, resulting in enhanced in vivo orthotopic and metastatic tumor growth (16). Consistent with previous studies on lung cancer $(17,18)$, downregulation of fibulin-3 expression was observed in the present study. These results suggest that fibulin-3 has dual functions as a positive and negative regulator of cancer cell growth depending on cell type. Wang et al (17) found that the frequency of methylation of the fibulin-3 gene promoter was significantly higher in NSCLC tissue samples than the frequency in the corresponding non-tumor tissue samples. Nomoto et al (11) found that the fibulin-3 gene was decreased in hepatocellular carcinoma (HCC) tumor tissue, and 24 of 48 HCC samples showed 
Table II. Correlation of the clinicopathological features of the NSCLC patients and serum levels of fibulin-3 using ELISA.

\begin{tabular}{|c|c|c|c|}
\hline $\begin{array}{l}\text { Clinicopathological } \\
\text { features }\end{array}$ & $\begin{array}{l}\text { Positive for } \\
\text { fibulin-3 } \\
\quad(n=18)\end{array}$ & $\begin{array}{l}\text { Negative for } \\
\text { fibulin-3 } \\
\quad(n=38)\end{array}$ & P-value \\
\hline Mean age (years) & $61.4 \pm 5.7$ & $64.3 \pm 5.8$ & 0.742 \\
\hline Gender & & & 0.294 \\
\hline Female & 8 & 10 & \\
\hline Male & 10 & 28 & \\
\hline Differentiation & & & 0.846 \\
\hline Well or moderate & 5 & 10 & \\
\hline Poor & 13 & 28 & \\
\hline Lymphatic invasion & & & 0.003 \\
\hline- & 5 & 28 & \\
\hline+ & 13 & 10 & \\
\hline Venous invasion & & & 0.512 \\
\hline- & 8 & 22 & \\
\hline+ & 10 & 16 & \\
\hline Histological type & & & 0.155 \\
\hline Squamous cell & 3 & 15 & \\
\hline Adenocarcinoma & 8 & 9 & \\
\hline Small cell & 7 & 14 & \\
\hline Tumor size $(\mathrm{cm})$ & & & 0.901 \\
\hline$<3$ & 11 & 21 & \\
\hline$\geq 3$ & 7 & 17 & \\
\hline pN category & & & 0.391 \\
\hline pNO & 4 & 8 & \\
\hline $\mathrm{pN} 1$ & 2 & 12 & \\
\hline $\mathrm{pN} 2$ & 6 & 8 & \\
\hline $\mathrm{pN} 3$ & 6 & 10 & \\
\hline Stage & & & 0.775 \\
\hline IIIB & 9 & 19 & \\
\hline IV & 9 & 19 & \\
\hline
\end{tabular}

NSCLC, non-small cell lung cancer.

promoter hypermethylation, in the 24 methylated cases. In the study of Tong et al (19), the fibulin-3 gene showed a higher methylation frequency in colorectal cancer (CRC) specimens while rare in non-cancer colorectal tissues, and its promoter methylation state was specifically associated with weak or absent expression of fibulin-3 protein. In the present study, we also confirmed that downregulation of fibulin-3 was caused by promoter hypermethylation. Notably, we did not find any association between fibulin-3 expression and the clinicopathological characteristics of the patients with stage IIB or IV NSCLC. However, in previous studies, downregulation of fibulin-3 was associated with advanced stage and lymph node metastasis in lung cancer (17) and CRC (19). We did find that circulating fibulin-3 was associated with lymph node metastasis. The discrepancy may be attributable to differences in the tumor entities examined and in the microenvironment of the different experimental settings.
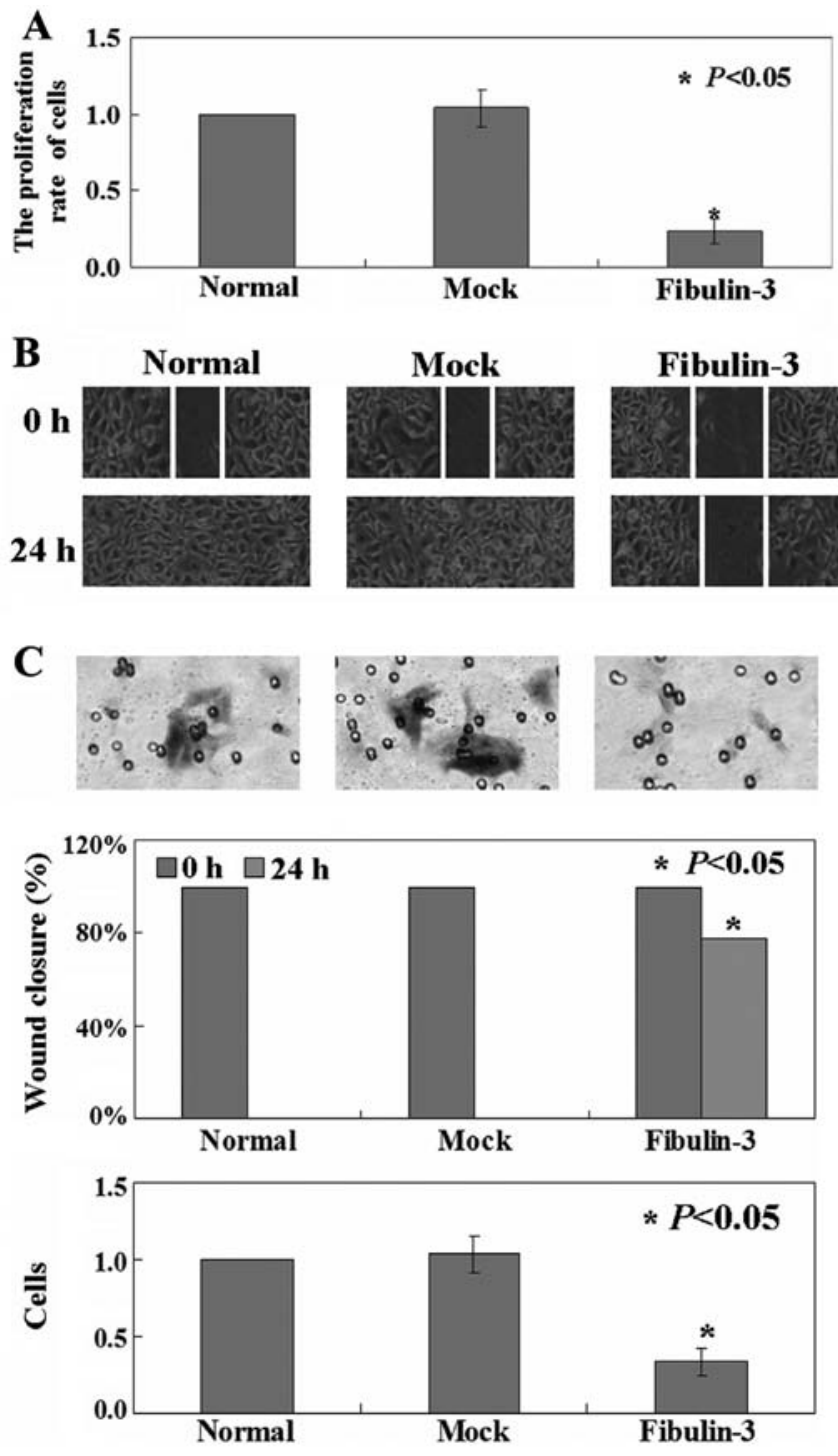

Figure 5. (A) The proliferation rate of A549 cells was determined by MTT assay. (B) Wound closure was quantified in the regions flanked by white lines. (C) The migratory capacity of cells was determined using Transwell assay. All results were statistically significant $\left({ }^{*} \mathrm{P}<0.05\right)$.

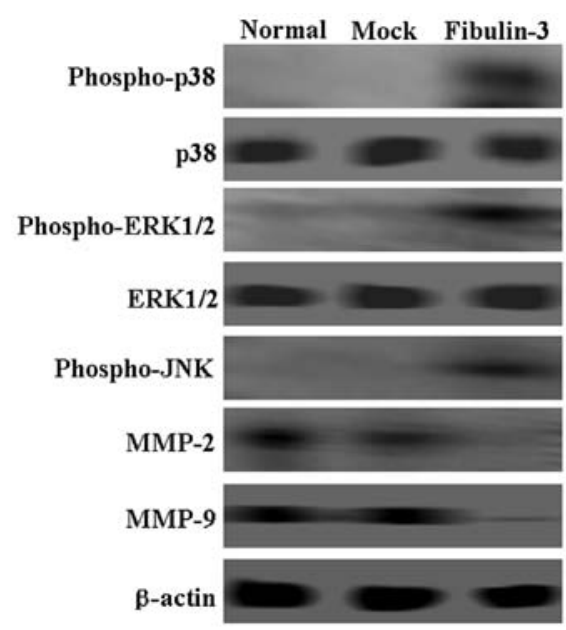

Figure 6. Levels of the phosphorylated forms of p38, ERK1/2 and JNK were increased in fibulin-3-positive cell lysates. However, the levels of total p38 and ERK1/2 remain unchanged. Decreased expression of MMP-2/-9 was confirmed by western blotting in fibulin-3-positive cell lysates. 
Circulating tumor cells (CTCs) captured from peripheral blood were recently used as a predictor for disease outcome and therapy response in cancer patients (20). Cytokeratinexpressing cells can be found in peripheral blood of advanced cancer patients but are rare in healthy donors (21). In the present study, we used the CellSearch ${ }^{\circledast}$ system which is the only US Food and Drug Administration authorized test for CTC enumeration in clinical practice to isolate CTCs (22). We found that fibulin-3 expression decreased the number of CTCs. The results indicate that upregulated expression of fibulin-3 can be used as a therapeutic strategy for NSCLC. In addition, levels of fibulin- 3 in plasma and effusions may aid in determining the diagnosis and prognosis of pleural mesothelioma (23). Our results were lacking healthy controls, thus we could not conclude that fibulin-3 is a marker for lung cancer. However, we confirmed that fibulin-3 may be used to indicate the progression of lung cancer. Circulating fibulin-3 level was correlated with favorable survival in total, stage IIIB and IV NSCLC patients. Previous studies also showed that reduced fibulin-3 expression was associated with worse prognosis of breast cancer (9), lung cancer (10) and hepatocellular carcinoma (11).

Both in our previous study (10) and the present study, fibulin-3 expression was revealed to be associated with the regulation of tumor cell growth and invasiveness in A549 cells by suppressing the expression of MMP-9 and -2 . Furthermore, the mechanism of fibulin-3 was identified by using western blotting. The positive regulation of phosphop38, phospho-ERK1/2 and phospho-JNK by fibulin-3 was shown in the present study. p38-MAPK was previously shown to regulate invasion by modulation of MMP-2/-9 mRNA levels and zymographic activity in bladder cancer model (24). p38-MAPK also modulated the inhibition of migration in $17 \beta$-estradiol-treated human colon cancer cells by inhibition of MMP-2-/9 expression (25). Similar to the present study, fibulin-3 inhibited invasion by upregulating p38-MAPK phosphorylation and downregulating the MMP-2/-9 protein expression in A549 cells.

Taken together, fibulin-3 downregulation is a common abnormality in lung cancer tissue and its expression is regulated by hypermethylation of the promoter region. Circulating fibulin-3 may play a role in tumor progression and the survival rate of patients with NSCLC. Our results suggest that fibulin-3 negatively modulates the invasiveness of lung cancer cells via regulation of p38-MAPK and MMP-2/9. In particular, fibulin-3 negatively controls the number of CTCs. These facts imply the diagnostic and therapeutic potential of fibulin-3 for NSCLC. Further research is needed prior to its clinical applications.

\section{Acknowledgements}

We are indebted to Li Yin for the plasmid pEGFP-C1-fibulin-3 and constructive suggestions in the preparation of this manuscript.

\section{References}

1. Jemal A, Bray F, Center MM, et al: Global cancer statistics. CA Cancer J Clin 61: 69-90, 2011.

2. Lam WK, White NW and Chan-Yeung MM: Lung cancer epidemiology and risk factors in Asia and Africa. Int J Tuberc Lung Dis 8: 1045-1057, 2004.
3. Kobayashi N, Kostka G, Garbe JH, et al: A comparative analysis of the fibulin protein family. Biochemical characterization, binding interactions, and tissue localization. J Biol Chem 282: 11805-11816, 2007.

4. Timpl R, Sasaki T, Kostka G and Ghu ML: Fibulins: a versatile family of extracellular matrix proteins. Nat Rev Mol Cell Biol 4: 479-489, 2003.

5. Pupa SM, Giuffré S, Castiglioni F, et al: Regulation of breast cancer response to chemotherapy by fibulin-1. Cancer Res 67: 4271-4277, 2007.

6. Yi CH, Smith DJ, West WW and Hollingsworth MA: Loss of fibulin-2 expression is associated with breast cancer progression. Am J Pathol 170: 1535-1545, 2007.

7. Albig AR and Schiemann WP: Fibulin-5 antagonizes vascular endothelial growth factor (VEGF) signaling and angiogenic sprouting by endothelial cells. DNA Cell Biol 23: 367-379, 2004.

8. Zhang Y and Marmorstein LY: Focus on molecules: fibulin-3 (EFEMP1). Exp Eye Res 90: 374-375, 2010.

9. Sadr-Nabavi A, Ramser J, Volkmann J, et al: Decreased expression of angiogenesis antagonist EFEMP1 in sporadic breast cancer is caused by aberrant promoter methylation and points to an impact of EFEMP1 as molecular biomarker. Int $\mathbf{J}$ Cancer 124: 1727-1735, 2009.

10. Yue W, Dacic S, Sun Q, et al: Frequent inactivation of RAMP2, EFEMP1 and Dutt1 in lung cancer by promoter hypermethylation. Clin Cancer Res 13: 4336-4344, 2007.

11. Nomoto S, Kanda M, Okamura Y, et al: Epidermal growth factorcontaining fibulin-like extracellular matrix protein 1, EFEMPI, a novel tumor-suppressor gene detected in hepatocellular carcinoma using double combination array analysis. Ann Surg Oncol 17: 923-932, 2010.

12. Lecka-Czernik B, Lumpkin CK Jr and Goldstein S: An overexpressed gene transcript in senescent and quiescent human fibroblasts encoding a novel protein in the epidermal growth factor-like repeat family stimulates DNA synthesis. Mol Cell Biol 15: 120-128, 1995.

13. Hata K, Dhar DK, Watanabe Y, et al: Expression of metastin and a G-protein-coupled receptor (AXOR12) in epithelial ovarian cancer. Eur J Cancer 43: 1452-1459, 2007.

14. Albig AR, Neil JR and Schiemann WP: Fibulins 3 and 5 antagonize tumor angiogenesis in vivo. Cancer Res 66: 2621-2629, 2006.

15. Hu B, Thirtamara-Rajamani KK, Sim H and Viapiano MS: Fibulin-3 is uniquely upregulated in malignant gliomas and promotes tumor cell motility and invasion. Mol Cancer Res 7: 1756-1770, 2009.

16. Seeliger H, Camaj P, Ischenko I, et al: EFEMP1 expression promotes in vivo tumor growth in human pancreatic adenocarcinoma. Mol Cancer Res 7: 189-198, 2009.

17. Wang R, Zhang YW and Chen LB: Aberrant promoter methylation of FBLN-3 gene and clinicopathological significance in non-small cell lung carcinoma. Lung Cancer 69: 239-244, 2010.

18. Kim EJ, Lee SY, Woo MK, et al: Fibulin-3 promoter methylation alters the invasive behavior of non-small cell lung cancer cell lines via MMP-7 and MMP-2 regulation. Int J Oncol 40: 402-408, 2012.

19. Tong JD, Jiao NL, Wang YX, Zhang YW and Han F: Downregulation of fibulin-3 gene by promoter methylation in colorectal cancer predicts adverse prognosis. Neoplasma 58: 441-448, 2011.

20. Cristofanilli M, Hayes DF, Budd GT, et al: Circulating tumor cells: a novel prognostic factor for newly diagnosed metastatic breast cancer. J Clin Oncol 23: 1420-1430, 2005.

21. Osta WA, Chen Y, Mikhitarian K, et al: EpCAM is overexpressed in breast cancer and is a potential target for breast cancer gene therapy. Cancer Res 64: 5818-5824, 2004.

22. Allard WJ, Matera J, Miller MC, et al: Tumor cells circulate in the peripheral blood of all major carcinomas but not in healthy subjects or patients with nonmalignant diseases. Clin Cancer Res 10: 6897-6904, 2004

23. Pass HI, Levin SM, Harbut MR, et al: Fibulin-3 as a blood and effusion biomarker for pleural mesothelioma. N Engl J Med 367: 1417-1427, 2012.

24. Hsu HH, Liu CJ, Shen CY, et al: p38 $\alpha$ MAPK mediates $17 \beta$-estradiol inhibition of MMP-2 and -9 expression and cell migration in human lovo colon cancer cells. J Cell Physiol 227: 3648-3660, 2012.

25. Kumar B, Koul S, Petersen J, et al: p38 mitogen-activated protein kinase-driven MAPKAPK 2 regulates invasion of bladder cancer by modulation of MMP-2 and MMP-9 activity. Cancer Res 70: 832-841, 2010. 